\title{
360 Degree Multi Sensor Fusion for Static and Dynamic Obstacles
}

\author{
Kai Schueler, Tobias Weiherer, Essayed Bouzouraa, and Ulrich Hofmann
}

\begin{abstract}
In this paper an approach for 360 degree multi sensor fusion for static and dynamic obstacles is presented. The perception of static and dynamic obstacles is achieved by combining the advantages of model based object tracking and an occupancy map. For the model based object tracking a novel multi reference point tracking system, called best knowledge model, is introduced. The best knowledge model allows to track and describe objects with respect to a best suitable reference point. It is explained how the object tracking and the occupancy map closely interact and benefit from each other. Experimental results of the $\mathbf{3 6 0}$ degree multi sensor fusion system from an automotive test vehicle are shown.
\end{abstract}

\section{INTRODUCTION}

\section{A. Motivation for 360 Degree Perception}

After the development of advanced driver assistance systems (ADAS) for the front area of the own vehicle like lane departure warning, adaptive cruise control (ACC), and collision warning, the side and the rear area came into focus, e.g. for obstacle detection in the blind spots. The next step is to combine the sensors of these ADAS in a multi sensor fusion system for a 360 degree environment perception. This is the base for future ADAS in complex scenarios like dense traffic jam, urban environments, intersections or for active safety systems with combined braking and evasion strategies.

Especially in the near environment of the own car it is important to detect the objects with dynamic states and dimensions. For example, in the scenario of a very close cut-in of another car in the own lane, the position of the front corner of the other car is important for the decision whether the own vehicle has to react upon it. Furthermore, the side of the nearest corner of the rear is relevant for the longitudinal and lateral automatic control of the own car.

\section{B. Related Work}

Over the past decade several object tracking approaches have been developed. In [1], [2], [3] a laser scanner is combined with a video sensor to perceive vehicles in the front area. In several cases the center of a bounding box is used for tracking, e.g. [2], [4]. In [2] a laser scanner defines a ROI for the video based object detection. In this case the initial object detection depends on both sensors. After the detection the laser scanner is also used for tracking. A cuboidal object shape model is introduced in [5] with different positions on the outer contour for the feature

K. Schueler and T. Weiherer are with the Faculty of Electrical Engineering, Lehrstuhl fuer Datenverarbeitung, Technische Universitaet Muenchen, Germany schueleretum. de, weihereretum.de

E. Bouzouraa and U. Hofmann are with the Advanced Driver Assistance Development Group, AUDI AG, Germany essayed.bouzouraadaudi.de, ulrich.hofmanneaudi.de

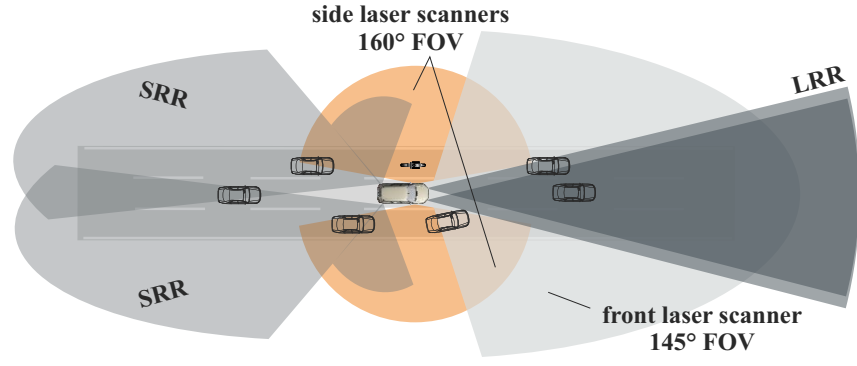

Fig. 1: Sensor Configuration

prediction. The state at the virtual rear axis is estimated. A similar multi reference point (RP) measurement model is used in [4] for associating laser scanner measurements and the position of the object's center point is estimated. In [6] a model based vehicle detection is performed by laser scan differencing and a particle filter is used for object state estimation. Due to the lack of observability of the object's center point, a virtual anchor point within a rectangular shape is tracked to overcome the problem of wrong velocities resulting from a moving center point.

In [7] and [8] the simultaneous localization and mapping (SLAM) problem was solved at the same time as the detection and tracking of moving objects (DATMO) by a combination of an occupancy map and object tracking. Dynamic objects are neither compensated nor object states are corrected in the occupancy map.

Our approach is an extension of the concept presented in [9]. The focus in this paper is on the 360 degree model based object tracking. The challenge to perceive objects from different viewing angles, for example, for the continuous tracking of an overtaking vehicle, is solved by using a multi RP model for tracking. Compared to [4], [5] different RPs are not used for the feature prediction but for the state estimation with respect to the best suitable RP. Furthermore, for the high level fusion, objects can be described with respect to different RPs. With this approach also the problem of velocities due to a moving object center is overcome because only measurable points on the object contour are measured and estimated.

\section{Sensor Configuration}

In order to fulfill the requirements of future ADAS, we need 360 degree multi sensor perception. Our chosen set of sensors is shown in Fig. 1. The sensor configuration consists of two long range radars (LRR) in the front, one front laser scanner, two side laser scanners, and two short range radars (SRR) at the rear side. The two LRR are, for example, used for ACC and the two SRR for blind spot detection in the 


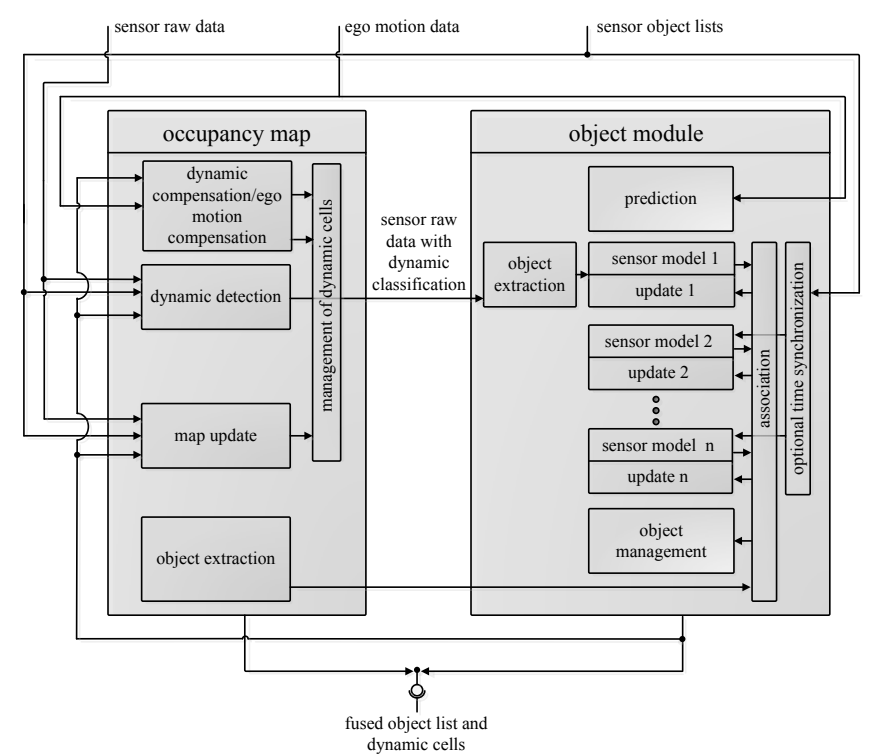

Fig. 2: Interaction between Occupancy Map and Object Module

current Audi A8. In principle, the remaining sensors are also suitable for automotive industrialization.

\section{Structure of the Paper}

In section II we explain our system architecture and how the object module and the occupancy map interact and benefit from each other. After this, in section III the model based object tracking is introduced. The focus in this section is on the best knowledge multi reference point tracking system. In section IV, experimental results of our 360 degree multi sensor fusion system from a test vehicle are presented.

\section{PERCEPTION SYSTEM ARCHITECTURE}

The object tracking presented in this work is part of a perception system composed of different experts. Each subsystem focuses on the description of a relevant part of the vehicle's environment using environment sensors and ego motion sensors. The perception modules interact with each other in order to generate a precise and consistent image of the environment (see Fig. 3) describing ego motion, objects, road infrastructure, free spaces, and unexplored areas. All consistent representations of all experts together are the internal representation of the real world. Using the dynamic models, a prediction of a dynamic scenario can be performed for short periods of time.

\section{A. Interaction between Occupancy Map and Model Based Object Tracking}

There are two main approaches to represent the vehicle's environment in order to realize ADAS. The first one consists in the model based object representation. In this case assumptions about the shape and the motion of the obstacles are made using shape and dynamic object models. The sensor measurements and the model knowledge are used to track the object states. This is a common way deployed by several actual driver assistance systems, e.g. ACC.

The occupancy grid map representation is a second method to describe the vehicle's environment. In general, sensor raw data are mapped into a two- or three-dimensional grid based data structure. During the mapping process the own vehicle motion is considered, and the sensor data are accumulated. This results in an accurate image of obstacles as well as free spaces and unexplored areas. Nevertheless, the grid map has a large memory consumption and a low level of abstraction which makes the interpretation of the map content difficult.

The quality of the object tracking and the occupancy map is enhanced by letting these two approaches interact and benefit from each other based on the concept presented in [9]. Fig. 2 demonstrates the interaction between both modules. The grid map is used to classify the sensor raw data according to their dynamic state. This is an important input for the object module which tracks only the moving objects since unstructured static obstacles are better represented by the grid map. Due to this, there is also the benefit that the number of false positive objects and wrong associations is lowered. Additionally, the state vector generated by the object tracking is deployed to compensate the position of moving objects on the map. This additional step in the mapping process has two major advantages. Firstly, the free space area within the map is extended. Secondly, moving objects can be represented by the occupancy map at the correct position. The resulting group of dynamic cells associated to an existing object describes the shape of objects (see Fig. 3). With the help of convenient correlation mechanisms, errors in the object state, estimated by the object tracker, can be detected and fed back to the object module. In this case the occupancy map is used as a virtual sensor.

\section{B. Laser Scan Point Dynamic Classification}

An important step for extracting dynamic objects out of laser measurements is to decide which laser measurements correspond to dynamic objects. This classification is accomplished in the occupancy map module and provided to the object module. In this section, the following mathematical notation will be used:

- $\mathrm{x}_{1: t}$ : Ego motion data until time $t$. As we build local occupancy maps, $\mathbf{x}_{t}$ describes the change of ego motion between $t-1$ and $t$.

- $\mathbf{z}_{1: t}$ : Sensor measurements (laser and radar) until $t$

- $\mathbf{m}_{t}$ : Occupancy map at time $t$

- $\mathbf{o}_{t}$ : Dynamic objects at time $t$

- $d_{t}^{k}$ : Binary random variable, which assigns the $k^{\text {th }}$ laser measurement a probability of corresponding to a dynamic object: $d_{t}^{k} \in\left\{\right.$ 'dyn' $\left.^{\prime},{ }^{\prime} s t a t^{\prime}\right\}$

In general, the mapping problem is given by estimating the probability [10]:

$$
P\left(\mathbf{m}_{t} \mid \mathbf{x}_{1: t}, \mathbf{z}_{1: t}\right)
$$

whereas the goal of object tracking can be formulated as:

$$
P\left(\mathbf{o}_{t} \mid \mathbf{x}_{1: t}, \mathbf{z}_{1: t}\right)
$$



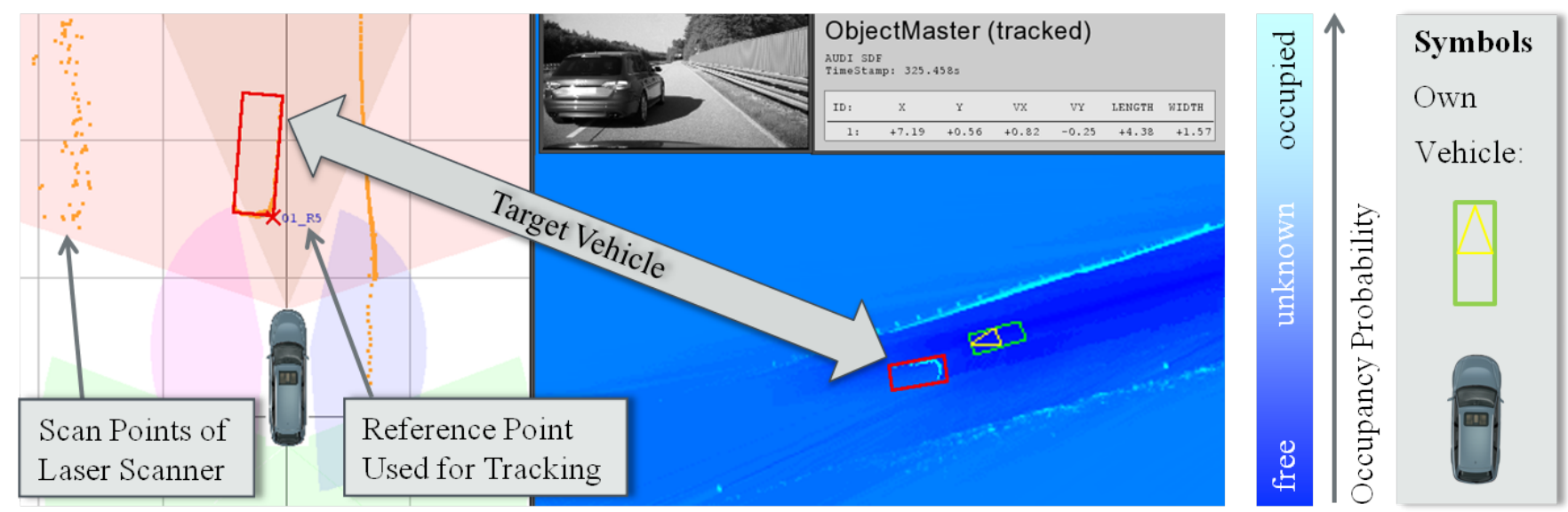

Fig. 3: Consistent Environment Representation from Object Module and Occupancy Map (Video Only for Documentation)

In our case of combined object tracking and grid mapping by a system of experts, additional information sources can be utilized for these estimations. On the one hand, the grid mapping algorithm should incorporate information about dynamic objects:

$$
\begin{array}{r}
P\left(\mathbf{m}_{t} \mid \mathbf{x}_{1: t}, \mathbf{z}_{1: t}\right)=\int P\left(\mathbf{m}_{t} \mid \mathbf{x}_{1: t}, \mathbf{z}_{1: t}, \mathbf{o}_{t}\right) . \\
\\
P\left(\mathbf{o}_{t} \mid \mathbf{x}_{1: t}, \mathbf{z}_{1: t}\right) d \mathbf{o}_{t}
\end{array}
$$

As already stated in the previous section, this is realized by compensating moving objects in the grid map. The detailed conditional probability distributions can be found in [9]. On the other hand, dynamic object tracking and extraction can be significantly simplified by consecutively introducing the binary random variables $d_{t}^{k}$ :

$$
\begin{array}{rl}
P\left(\mathbf{o}_{t} \mid \mathbf{x}_{1: t}, \mathbf{z}_{1: t}\right)=\sum_{d_{t}^{k}} & P\left(\mathbf{o}_{t} \mid \mathbf{x}_{1: t}, \mathbf{z}_{1: t}, d_{t}^{k}\right) \\
& \left.P\left(d_{t}^{k} \mid \mathbf{x}_{1: t}, \mathbf{z}_{1: t}\right)\right) \\
= & P\left(\mathbf{o}_{t} \mid \mathbf{x}_{1: t}, \mathbf{z}_{1: t}, d_{t}^{k}=^{\prime} d y n^{\prime}\right) \\
& P\left(d_{t}^{k}=^{\prime} d y n^{\prime} \mid \mathbf{x}_{1: t}, \mathbf{z}_{1: t}\right)+ \\
& P\left(\mathbf{o}_{t} \mid \mathbf{x}_{1: t}, \mathbf{z}_{1: t}, d_{t}^{k}=^{\prime} s_{a t}\right) . \\
& P\left(d_{t}^{k}=^{\prime} s^{\prime} t a t^{\prime} \mid \mathbf{x}_{1: t}, \mathbf{z}_{1: t}\right)
\end{array}
$$

The dynamic classification of a single laser measurement can be reformulated by using the past grid map $\mathbf{m}_{t-1}$ :

$$
\begin{aligned}
P\left(d_{t}^{k}={ }^{\prime} d y n^{\prime}\right. & \left.\mid \mathbf{x}_{1: t}, \mathbf{z}_{1: t}\right) \\
= & \sum_{\mathbf{m}_{t-1}}\left(P\left(d_{t}^{k}=^{\prime} d y n^{\prime} \mid \mathbf{x}_{1: t}, \mathbf{z}_{1: t}, \mathbf{m}_{t-1}\right) .\right. \\
& \left.P\left(\mathbf{m}_{t-1} \mid \mathbf{x}_{1: t}, \mathbf{z}_{1: t}\right)\right) \\
= & \sum_{\mathbf{m}_{t-1}}\left(P\left(d_{t}^{k}=^{\prime} d y n^{\prime} \mid \mathbf{x}_{t}, \mathbf{z}_{t}, \mathbf{m}_{t-1}\right) .\right. \\
& \left.P\left(\mathbf{m}_{t-1} \mid \mathbf{x}_{1: t-1}, \mathbf{z}_{1: t-1}\right)\right)
\end{aligned}
$$

The full a posteriori estimation of this density function is intractable as we do, for example, not maintain a probability distribution over several possible grid maps $\mathbf{m}_{t-1}$. Consequently, we use the last maximum likelihood map and simplify $P\left(d_{t}^{k}={ }^{\prime} d y n^{\prime} \mid \mathbf{x}_{t}, \mathbf{z}_{t}, \mathbf{m}_{t-1}\right)$ to estimate a binary classification whether a laser measurement belongs to a dynamic object or not. Using this simplification, the consideration of all laser measurement classifications results in:

$$
\begin{aligned}
& P\left(\mathbf{o}_{t} \mid \mathbf{x}_{1: t}, \mathbf{z}_{1: t}\right) \\
& \quad=P\left(\mathbf{o}_{t} \mid \mathbf{x}_{1: t}, \mathbf{z}_{1: t}, d_{t}^{1}=^{\prime} d y n^{\prime}, \ldots, d_{t}^{k}=^{\prime} d y n^{\prime}\right)
\end{aligned}
$$

The different sources for estimating the dynamic classification of a single laser measurement are described in the following.

1) Radar measurements: By utilizing the Doppler principle, radar sensors provide reliable data about the radial velocity of measured objects. On the downside, radar sensors typically only provide a single radar reflection center. In order to use these measured objects for several neighboring laser measurements, the uncertainties of a radar measurement can be modeled by a two-dimensional Gaussian distribution [9]. After synchronizing the laser and radar measurements, the binary information about the existence of dynamic and static radar ellipses can be stored in separated map layers. In this way, only a small amount of extra memory is required and radar measurements from the past can be used.

2) Dynamic object prediction: Additional information about the correspondence of laser measurements and dynamic objects can be gained from tracked dynamic objects. By using a grid map $\mathbf{m}_{t}$ with associated dynamic objects, cells corresponding to moving objects can be predicted and used to match and classify laser measurements.

3) Occupancy Grid Map: Finally, a well-known idea for classifying laser measurements is to find differences between raw laser sensor data and occupancy maps built from past measurements [7], [11]. We enhance these algorithms to assure a robust detection. First, we model the defocusing of the laser sensor beam's field of view, resulting in small measurement lines instead of single reflection points. Second, we extend these line regions by a dilatation of a binary image to overcome inaccuracies resulting from ego, sensor data noise, and discretization errors [9]. After the projection of laser data on the map, the occupancy values of the resulting regions are compared to predefined threshold values and classified as dynamic or static. 


\section{MODEL BASED OBJECT TRACKING}

For the model based multi target object tracking the philosophy is used that each sensor contributes to the environment perception as best as it can. In order to exploit the capabilities of each sensor as good as possible, each sensor has been analyzed in detail in real world scenarios with a reference system consisting of a differential global positioning system (DGPS) and an inertial platform before the sensors are deployed in the object tracking. The acquired knowledge of the sensor behaviour is used in a sensor model in our object tracking module.

\section{A. Object Representation - Best Knowledge Model}

In our multi sensor system the sensors should represent their "best knowledge" of the static and dynamic environment avoiding unnecessary assumptions for length and width and give it to the responsible expert modules for fusion. For dynamic objects this is the object module. How should this "best knowledge" representation look like?

1) It should be able to represent object lists, which only consist of a relative position and speed in $\mathrm{x}$ and $\mathrm{y}$ with uncertainty in position, speed, and moving direction, but no dimensions of the object.

2) The representation should also be able to describe the bounding box of vehicles with length, width, and optional height. In dependence of the mounting positions of the sensors at the rear, side and front and their observation viewing angles, the same object may appear from different views in the different sensors. We want that each sensor is able to represent its best knowledge about the measured object and, therefore, it has to choose the coordinate system, in which the values and uncertainties of the measured sensor data are best described. We give the sensors the ability to choose the origins of the possible local object coordinate systems from nine reference points (RP), see Fig. 4. The object state at the RP is then described relatively to the own vehicle coordinate system whereas the RP is part of the description. If the speed is unknown, there could be four suitable consistent representations. Two have the only difference of $\pi$ in the orientation. If it does not matter for the tracking if a car is driving backwards or if it is oncoming traffic, this ambiguity can be solved by limiting $\Psi$ to $(\pi,-\pi]$. The second ambiguity results if it cannot be determined whether the length or the width of an object is measured. These ambiguities have to be solved either in the sensor specific association in the tracking phase or by the fusion in the initial phase. For the radar sensors one of the results of the sensor analysis phase is which RP describes best the measurements depending on the situation.

3) The representation can be extended by further information about the object, e.g. special features like color, position of rear or front lights, texture patterns, history and statistics of data and other contour details, where it is necessary. This additional information is represented

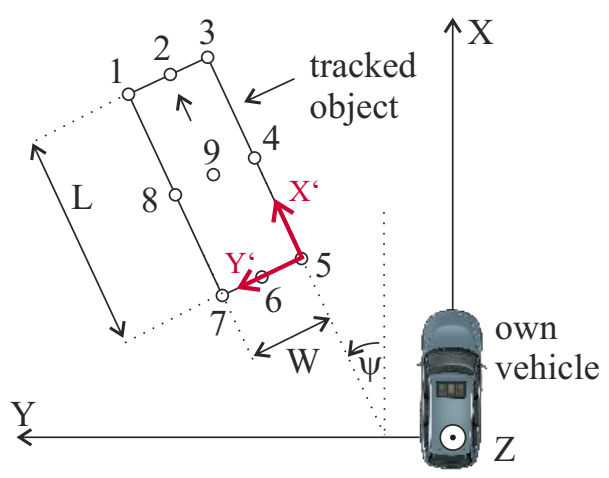

Fig. 4: Multi RP Object Representation with RP 5 Selected as Center of the Local Object Coordinate System

in the local object coordinate system. The goal is to get a compact and precise representation with all relevant information for tracking.

1) Reference Point Transformation: In the case of 360 degree perception objects are seen from different viewing angles. In our approach the state of an object is estimated with respect to the best observable RP which changes over time as illustrated in Fig. 5. How to transform the description of either measured sensor objects or of tracked objects with respect to a new RP is shown in the following.

Let $\mathbf{d}$ be the description at the current RP and $\mathbf{y}$ be the description at the new RP. $\mathbf{P}_{\text {in }}$ and $\mathbf{P}_{\text {out }}$ are the respective error covariance matrices. All quantities are described relatively to the own vehicle coordinate system $((X, Y)$ in Fig. 4). $\mathbf{d}^{T}$ equates to:

$$
\mathbf{d}^{T}=\left(x, y, v_{x}, v_{y}, a_{x}, a_{y}, \Psi, L, W\right)
$$

The lever vector $\mathbf{k}^{\prime}$ is the position change from the current to the new RP and has to be considered in the local object coordinate system for the RP transformation. For a transformation from RP 5 to $2 \mathbf{k}^{\prime T}$ is equal to:

$$
\mathbf{k}^{\prime T}=(L, 0.5 \cdot W) ; \mathbf{r}^{\prime T}=\left(\mathbf{k}^{\prime T}, 0\right)
$$

Looking from the own vehicle coordinate system at a rotating target object results in different velocities and accelerations at the different RPs. With the angular velocity vector $\omega$ and a three-dimensional lever vector $\mathbf{r}^{\prime}$ the resulting tangential velocity $\mathbf{v}_{t}$ is given by $\mathbf{v}_{t}=\omega \times \mathbf{r}^{\prime}$ and the acceleration due to the centripetal acceleration is equal to $\mathbf{a}_{c}=\omega \times\left(\omega \times \mathbf{r}^{\prime}\right)$ [12]. In our case the velocity change due to the rotation described in the local object coordinate system can be determined to:

$$
\mathbf{v}^{\prime}{ }_{t}=\left(\begin{array}{c}
0 \\
0 \\
\dot{\Psi}
\end{array}\right) \times\left(\begin{array}{c}
k_{1}^{\prime} \\
k_{2}^{\prime} \\
0
\end{array}\right)=\left(\begin{array}{c}
-\dot{\Psi} k_{2}^{\prime} \\
\dot{\Psi} k_{1}^{\prime} \\
0
\end{array}\right)
$$

The acceleration change $\mathbf{a}^{\prime}{ }_{c}$ in the local object coordinate system equates to:

$$
\mathbf{a}_{c}^{\prime}=\left(\begin{array}{c}
0 \\
0 \\
\dot{\Psi}
\end{array}\right) \times \mathbf{v}^{\prime}{ }_{t}=\left(\begin{array}{c}
-\dot{\Psi}^{2} k_{1}^{\prime} \\
-\dot{\Psi}^{2} k_{2}^{\prime} \\
0
\end{array}\right)
$$


The determined position, velocity, and acceleration changes have to be transformed from the local object coordinate system to the own vehicle coordinate system due to the different orientation of the coordinate systems. The rotation matrix around the Z-Axis in the $\mathrm{X}-\mathrm{Y}$ plane for a twodimensional vector is given by:

$$
\mathbf{R}_{z}=\left(\begin{array}{cc}
\cos (\Psi) & -\sin (\Psi) \\
\sin (\Psi) & \cos (\Psi)
\end{array}\right)
$$

The description at the new RP equates to:

$$
{ }^{6} \mathbf{y}={ }^{6} \mathbf{d}+\left(\begin{array}{c}
\mathbf{R}_{z} \cdot \mathbf{k}^{\prime} \\
\mathbf{R}_{z} \cdot{ }^{2} \mathbf{v}^{\prime}{ }_{t} \\
\mathbf{R}_{z} \cdot{ }^{2} \mathbf{a}^{\prime}{ }_{c}
\end{array}\right)
$$

whereas ${ }^{i} \mathbf{y}$ describes a vector with the first $i$ elements of y. ${ }^{i} \mathbf{y}={ }^{i} \mathbf{d}$ for $i=7,8,9$. Due to the RP change, the error covariance matrix has to be recalculated. With the jacobian matrix $\mathbf{J}=\partial \mathbf{y} / \partial \mathbf{d}$ and $\mathbf{D}=\partial \mathbf{y} / \partial(L, W)$ and the lever factors error covariance matrix:

$$
\begin{gathered}
\mathbf{P}_{\text {lever }}=\left(\begin{array}{cc}
\sigma_{L}^{2} & 0 \\
0 & \sigma_{W}^{2}
\end{array}\right) \\
\mathbf{P}_{\text {out }}=\mathbf{J} \cdot \mathbf{P}_{\text {in }} \cdot \mathbf{J}^{T}+\mathbf{D} \cdot \mathbf{P}_{\text {lever }} \cdot \mathbf{D}^{T}
\end{gathered}
$$

\section{B. Object Estimation, Detection, and Association}

Our object tracking module utilizes a Kalman Filter (KF). All objects are tracked relatively to the own vehicle. In our sensor set (see Fig. 1) the laser scanners trigger a filter cycle because they have the shortest cycle time and the lowest latency. This is the time period from sensor measurement time until the data arrive in our development framework. Furthermore, compared to the raw laser measurements without dynamic information, the objects from the radar sensors can be more easily predicted to the laser measurement time. If a new laser scanner measurement arrives, all sensor data from the radar sensors received since the last laser scanner measurement are predicted to the measurement time of the laser scanner. After this, the internal representation of the object module is predicted to the laser scan measurement time. For all sensors the expected features, which should be measured by the sensor, are generated. These features are associated with the measured or preprocessed sensor features and the internal representation is updated, respectively. In our current implementation all sensor data is preprocessed up to bounding box level. The radar scanners provide directly object lists on bounding box level.

The dynamic classified laser scan points from the occupancy map are preprocessed in a sensor model. We use an adaptive breakpoint [13] approach to extract groups of laser scan points which are likely to belong to the same surface. Afterwards, corners are detected with an extended iterative endpoint fit (IEPF) [13] algorithm. From the extracted polygonal lines total least squares regression lines are estimated with an eigenvector line fitting [14] approach. From these extracted lines and the dynamic information from the occupancy map, objects are created. With respect to the own vehicle coordinate system, pairs of concave lines are discarded because these do not describe a valid object fitting our shape model.

In the association of the object module, gating combined with a global nearest neighbor approach (GNN) [15] based on either the euclidean distance or the Mahalanobis Distance [10] is used.

\section{Kalman Filter}

The object module estimates all quantities at a RP relatively to the cartesian own vehicle coordinate system with exception of the yaw rate influence of the own vehicle because the own yaw rate is well known from the ego motion data. First experiments have shown that if the yaw rate influence of the own vehicle is not decoupled, track loses occur more often. The position $(x, y)$, velocity $\left(v_{x}, v_{y}\right)$, acceleration $\left(a_{x}, a_{y}\right)$, yaw angle $(\Psi)$, and steering angle $(\lambda)$ are estimated by a KF. The width $(W)$ and the length $(L)$ are rule based innovated. For example, the whole length is only visible in certain situations and should be kept as long as it is reliable and no sensor measures something contradictory. Thus, the state vector $\mathbf{x}$ equates to:

$$
\mathbf{x}^{T}=\left(x, y, v_{x}, v_{y}, a_{x}, a_{y}, \Psi, \lambda\right)
$$

As shown in [16], a simplified approximation for the yaw rate can be derived from the single-track model assuming a negligible side slip angle. With the target vehicle variables $\lambda$ being the steering angle and $d_{a}$ being the distance between the wheel axles, the yaw rate can be approximated to:

$$
\dot{\Psi}=\frac{|v|}{d_{a}} \cdot \lambda
$$

The unknown steering angle $\lambda$ is assumed to be constant with $\dot{\lambda}$ as random noise. Equation (16) ensures that a vehicle can only have a yaw rate if it moves. The fact that $d_{a}$ cannot be measured in most cases does not matter because it just results in a linear scaling of the estimated steering angle $\lambda$. Thus, $d_{a}$ can be set to a value of an average car. The approximation is used for the dynamic model of the yaw angle:

$$
\Psi_{k}=\Psi_{k-1}+\frac{|v|}{d_{a}} \cdot \lambda \cdot \Delta T
$$

whereas $\Delta T$ is the time difference between two filter cycles. For the position, velocity, and acceleration a constant acceleration model is used. The yaw rate influence of the own vehicle is compensated directly after the prediction step. Let $\Delta \Psi_{\text {own }}$ be the own vehicle yaw angle difference between two filter cycles due to the own vehicle yaw rate described in the own vehicle coordinate system at time step $k$. The position, velocity, and acceleration vectors of all target objects are rotated by $\Delta \Psi_{\text {own }}$ and $\Delta \Psi_{\text {own }}$ is added to the yaw angles of all target objects.

\section{EXPERIMENTAL RESULTS}

Fig. 3 shows the resulting consistent environment representation of our occupancy map and our object module. In Fig. 5 the applied "best knowledge" model is illustrated. The meaning of the symbols is explained in Fig. 3. 


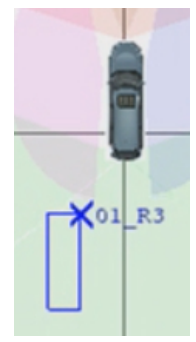

(a)

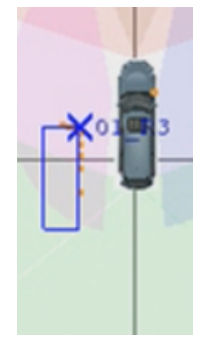

(b)

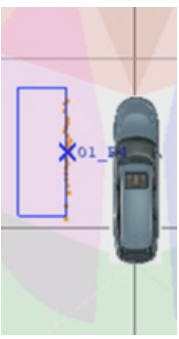

(c)

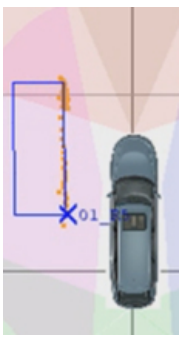

(d)

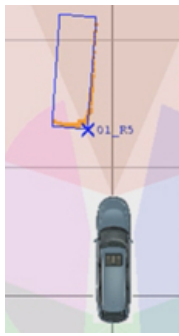

(e)

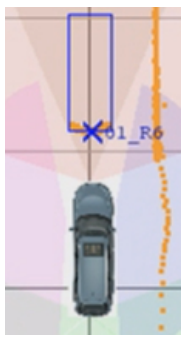

(f)

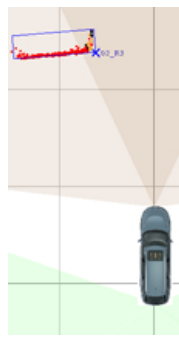

(g)

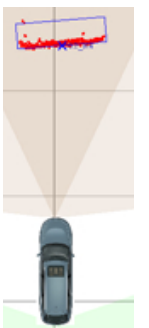

(h)

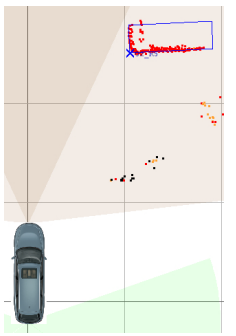

(i)

Fig. 5: Fusion Results of Overtaking Scenario with Close Cut-In, (a)-(f), and Cross Traffic at a T Junction (g)-(i)

In Fig. 5c RP 4 is chosen because the sensor analysis has shown that the sensor has difficulties to detect the corners of closely overtaking cars if the reflection angles of the laser beams are acute. In this case the measurement is interpreted with higher uncertainty in $x$-position. In Fig. 5d first an update with RP 3 from the front laser and afterwards an update with RP 5 from the side laser is performed. In this case the front laser scanner can only observe the obstacle's front right corner. From the side laser scanner's point of view, the object is leaving the FOV and, therefore, the rear right corner can best be measured. In Fig. 5g - 5i the own vehicle is standing at a $\mathrm{T}$ junction and a vehicle is crossing the front laser's FOV from left to right. First the front right side is best observed. In the middle of the FOV, Fig. 5h, the middle of the right side is the suitable choice. In Fig. $5 \mathrm{i}$ an L-shape is measured which is described by RP 5 .

\section{A. Execution Time}

For our sensor setup the complete computation time per processing cycle of the occupancy map and the object module is on average $5 \mathrm{~ms}$ on a Core i7 m640 laptop with 2.8 $\mathrm{GHz}$, using only one core. This was measured on a sequence including multiple objects with a duration of several minutes. The occupancy map has a size of $140 \mathrm{~m} \times 140 \mathrm{~m}$ and a grid cell size of $0.2 \mathrm{~m}$. The object module is limited to track at maximum 80 objects.

\section{CONCLUSIONS AND FUTURE WORK}

\section{A. Conclusions}

In this paper we have presented an approach for 360 degree multi sensor fusion for static and dynamic obstacles combining the advantages of an occupancy map and model based object tracking. The introduced best knowledge model enables multi sensor tracking of objects, including dimensions, from different viewing angles.

\section{B. Future Work}

In future work we will focus on extending the dynamic models used in the KF. In order to easily model different dynamic behaviours and to improve the ability to predict dynamic scenes, the movement of the ego vehicle and the target objects will be further decoupled. Furthermore, the focus will be intensified on using the map feedback for correcting the object states. Additionally, we will expand the best knowledge model to enable the sensor to represent the reliability of the chosen RP and alternative RPs.

\section{REFERENCES}

[1] N. Kaempchen, K. Fuerstenberg, A. Skibicki, and K. Dietmayer, "Sensor fusion for multiple automotive active safety and comfort applications," Advanced microsystems for automotive applications 2004, p. 137163, 2004.

[2] S. Wender and K. Dietmayer, "3d vehicle detection using a laser scanner and a video camera," Intelligent Transport Systems, IET, vol. 2, no. 2, p. 105112, 2008.

[3] M. Munz, K. Dietmayer, and M. Mahlisch, "A sensor independent probabilistic fusion system for driver assistance systems," in Intelligent Transportation Systems, 2009. ITSC'09. 12th International IEEE Conference on, 2009, p. 16.

[4] S. Wender, "Multisensorsystem zur erweiterten Fahrzeugumfelderfassung," Ph.D. dissertation, Univ., Fak. fr Ingenieurwiss. und Informatik, 2008.

[5] N. Kaempchen, "Feature level fusion of laser scanner and video data for advanced driver assistance systems," Ph.D. dissertation, Ulm, 2007.

[6] A. Petrovskaya and S. Thrun, "Model based vehicle detection and tracking for autonomous urban driving," Autonomous Robots, vol. 26, no. 2, p. 123139, 2009.

[7] C.-C. Wang and C. Thorpe, "Simultaneous localization and mapping with detection and tracking of moving objects," in Robotics and Automation, 2002. Proceedings. ICRA 'O2. IEEE International Conference on, vol. 3, 2002, pp. $2918-2924$.

[8] C. Wang, C. Thorpe, and A. Suppe, "Ladar-based detection and tracking of moving objects from a ground vehicle at high speeds," in Intelligent Vehicles Symposium, 2003. Proceedings. IEEE, 2003, p. 416421.

[9] M. E. Bouzouraa and U. Hofmann, "Fusion of occupancy grid mapping and model based object tracking for driver assistance systems using laser and radar sensors," in 2010 IEEE Intelligent Vehicles Symposium (IV). IEEE, Jun. 2010, pp. 294-300.

[10] S. Thrun, W. Burgard, and D. Fox, Probabilistic Robotics. The MIT Press, Aug. 2005.

[11] T.-D. Vu, O. Aycard, and N. Appenrodt, "Online localization and mapping with moving object tracking in dynamic outdoor environments," in Intelligent Vehicles Symposium, 2007 IEEE, june 2007, pp. 190 -195 .

[12] H. Goldstein, C. P. Poole, and J. L. Safko, Classical Mechanics, 3rd ed. Addison Wesley, Jun. 2001.

[13] G. A. Borges and M. J. Aldon, "Line Extraction in 2D Range Images for Mobile Robotics," Journal of Intelligent and Robotic Systems, vol. 40, no. 3, p. 267297, 2004.

[14] J. A. Mieghem, H. I. Avi-Itzhak, and R. D. Melen, "Straight line extraction using iterative total least squares methods," Journal of Visual Communication and Image Representation, vol. 6, no. 1, p. 5968, 1995.

[15] P. Konstantinova, A. Udvarev, and T. Semerdjiev, "A study of a target tracking algorithm using global nearest neighbor approach," in Proceedings of the International Conference on Computer Systems and Technologies (CompSysTech03), 2003.

[16] B. Mysliwetz, "Parallelrechner-basierte Bildfolgen-Interpretation zur autonomen Fahrzeugsteuerung," Ph.D. dissertation, Universitaet der Bundeswehr Muenchen, 1990. 